Errata

\title{
Vertical eye movements related signals in antidromically identified medullary reticular formations neurons in the altert cat
}

\author{
J.M. Delgado-García, P.P. Vidal, C. Gómez, and A. Berthoz \\ Exp Brain Res (1988) 70: 585-589
}

There were three fairly serious omissions in the above article: part of the text was left out on three different pages.

The text should read as follows:

On p. 639 (left column, four lines from bottom):

Semi-thin $(0.5 \mu \mathrm{m}$ thick) sections were stained with toluidine blue. Semi-serial ultrathin sections through the grafts were stained with uranyle acetate and lead citrate and were examined using a Philips 301 electron microscope.
On p. 642 (left column, paragraph 2, line 1):

Similar types of chromaffin cells were found in cell grafts. Most of the transplanted chromaffin cells were noradrenaline-phenotype cells, as seen in the normal adrenal medulla.

On p. 645 (right column, paragraph 2, line 18):

This may imply that denervation of the adrenal gland can result in the appearance of new chromaffin cell types.

\section{Toxic effects of triethyldodecylammoniumbromide (TEA-C ${ }_{12}$ ) on myelinated nerve fibers and blood-nerve barrier in the mouse}

\author{
R.J. Seitz, P. Lipfert, A. Willrich, and F. Himmelmann
}

Exp Brain Res (1989) 74: 293-302

Due to an error in the lay-out, Fig. 4a-f has been printed in an earlier, unrevised version. The correct figure is as follows:

Also, the figure legend of Fig. 5 was printed incomplete.

It should read in addition: Fig. 5a and b. Bar $5 \mu \mathrm{m}$. 